\title{
Effects of Inlet Types and Lengths on the Flow Field of Cyclone Separators
}

\author{
S. Y. $\mathrm{Wu}^{1 \dagger}$, Y. W. Xiao ${ }^{1}$, C. D. $\mathrm{Nie}^{2}$ and Z. M. $\mathrm{Wu}^{3}$ \\ ${ }^{1}$ School of Mechanical Engineering, Xiangtan University, Xiangtan 411105, China. \\ ${ }^{2}$ School of Energy Science and Engineering, Central South University, Changsha, Hunan 410083, China \\ ${ }^{3}$ School of Chemical Engineering, Xiangtan University, Xiangtan 411105, China \\ †Corresponding Author Email: wusy2015@xtu.edu.cn
}

(Received March 30, 2021; accepted October 10, 2021)

\begin{abstract}
The effect of inlet type and length on the flow field was considered computationally for seven cyclone separators. The turbulent model was described by the Reynolds Stress Model (RSM). The air-water interface in underflow pipe and the spatial distribution of particles were tracked by the Volume of Fluid (VOF) model and Discrete Phase Model (DPM), respectively. Comparison investigations showed that inlet type and length had important impacts on flow field of cyclone. The instability flow field and back mixing phenomena were eliminated in symmetric double-inlet cyclone. The turbulent dissipation is obvious with a short inlet length. When it increased to $1.25 \mathrm{D} / 2$, the area and intensity of the turbulent dissipation tended to be stable. The optimum cyclone is the symmetric double-inlet with inlet length of $1.25 \mathrm{D} / 2$. When the particle diameter was larger than $5 \mu \mathrm{m}$, the complete separation could be realized.
\end{abstract}

Keywords: Cyclone; Inlet type; Inlet length ;Flow field.

\section{NOMENCLATURE}

$\begin{array}{llll}a & \text { inlet wide } & P_{\mathrm{ij}} & \text { stress production } \\ b & \text { inlet height } & \operatorname{Re}_{\mathrm{p}} & \text { particle Reynolds number } \\ \mathrm{c} & \text { inlet length } & S & \text { immersion length } \\ \mathrm{C}_{\mathrm{D}} & \text { drag coefficient } & t & \text { time } \\ d & \text { outlet diameter } & \mathrm{u} & \text { fluid phase velocity } \\ d_{1} & \text { finder diameter } & \overline{\mathrm{u}} & \text { mean velocity } \\ \mathrm{d}_{\mathrm{p}} & \text { particle diameter } & u_{\mathrm{p}} & \text { particle velocity } \\ D & \text { cylinder diameter } & x_{\mathrm{i}} & \text { position } \\ D_{\mathrm{T}}, \mathrm{ij} & \text { stress diffusion } & \alpha & \text { volume fraction } \\ F_{\mathrm{D}} & \text { drag force } & \varepsilon_{\mathrm{ij}} & \text { dissipation } \\ \mathrm{g} & \text { gravitational acceleration } & \eta & \text { separation efficiency } \\ H_{1} & \text { outlet height } & \mu & \text { molecular viscosity of fluid } \\ H_{3} & \text { cylinder height } & \rho & \text { fluid density } \\ \dot{m}_{\mathrm{p}} & \text { flow rate of the particles } & \rho_{\mathrm{g}} & \text { particle density } \\ N & \text { particle number } & \varphi_{\mathrm{ij}} & \text { pressure strain }\end{array}$

\section{INTRODUCTION}

Cyclone separator is a device that separates twophase flow using centrifugal force and has a unique two-layer vortex feature. Due to its simple geometric structure, low operating and maintenance costs, high durability and simple operation, the cyclone separator is widely used in various industries including boiler flue gas treatment, indoor air purification and drug purification (Rafiee and Sadeghiazad 2016; Pan et al. 2021; Caliskan et al. 2019). Optimizing the structural size to improve the separation efficiency of the cyclone separator, 
its application field is expected to be further expanded.

The gas-liquid two-phase flow behaviors are extremely complex in a cyclone separator. The structural size plays an important role on the flow field and the separation performance of the cyclone (Gao et al. 2020; Nardo et al. 2018). Computational Fluid Dynamics (CFD) is an effective technique to explore the complex flow field in a cyclone, and predict the droplet behavior and performance. The effects of cyclone inlet dimensions on the flow pattern and performance were numerically studied (Elsayed and Lacor 2011)(Yao et a. 2021). Keeping inlet velocity of gas and mean particle diameter constant, the variety of particle trajectories and cyclone efficiency was studied along with varying coefficient of restitution and particle size (Prabhansu et al. 2017). Different inlet angles had a great influence on the separate performance and pressure drop of the cyclone separator (Wang et al. 2019; Wasilewski and Brar 2019; Misiulia et al. 2015 ). 20 bend angles of the inlet duct of cyclone separators were studied by CFD (Wasilewski and Brar 2019). The angle of the inlet duct bend was found to significantly affect the Euler number, with marginal effects on the Stokes number. 10 cyclones with different inlet dimensions were investigated and increasing the inlet angle could reduce tangential velocity and pressure drop (Misiulia et al. 2015). The cyclone geometry was optimized to minimize pressure drop and $78 \%$ saving in the pressure drop was realized for a new optimal cyclone design (Elsayed 2015). The design of reducing pressure drop holes is proposed (Prasannaa et al. 2021).

In some applications, the cyclone is placed in the container to avoid the back-mixing and feed blocking phenomenon, called the built-in cyclone (Wright et al. 2013; Bao et al. 2017). In this case, the shape and size of the cyclone is limited by the outer container and different from conventional cyclones. The built-in cyclone was invented to provide pre-classified activated catalyst (Wright et al. 2013). A recycling supersonic separator with two kinds of built-in cyclone was proposed to improve the efficiency of separation and dehydration (Bao et al. 2017). The length of vertical tube should be considered to avoid the gas getting into a cyclone to affect the separation of solid particles (Kuznetsov and Shchipko 2010). A built-in cyclonic water filter was designed to absorb dust and purify the air (Wang 2004). The renewed application of cyclone separator leads to an increased interest in its design. The inlet type and length are two important parameters in the built-in cyclone (Prabhansu et al. 2017).

In the present work, four different inlet types of the cylinder have constructed, namely: single inlet, symmetric double-inlet with two different dimensions, up-down double-inlet. Meanwhile, the inlet length is considered. The main purpose is to reveal the effect of the inlet type and inlet length on the flow field based on the flow property, flow stability and secondary flow.

\section{NUMERICAL METHOD}

\subsection{Configuration of cyclone}

Figure 1 shows the cyclone geometry, which is described by the following parameters, the inlet wide $a$, height $b$ and length $c$, the vortex finder diameter $d_{1}$ and immersion length $S$, the cylinder height $H_{3}$ and diameter $D$, the underflow outlet diameter $d$ and height $H_{1}$, the cyclone total height $H_{\mathrm{t}}$. These constant characteristic parameters are listed in Table 1.

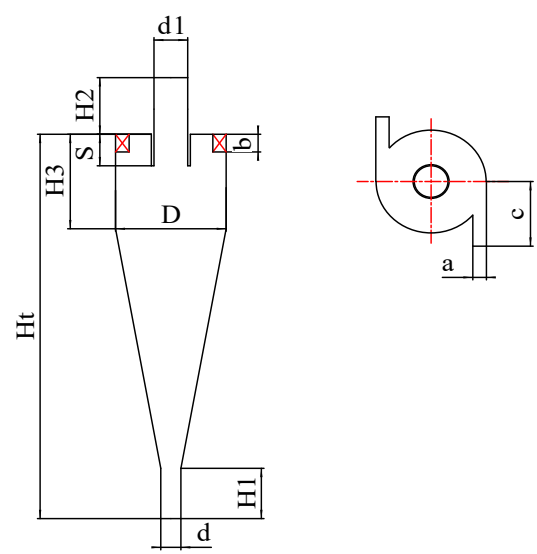

Fig. 1. Geometric structure for cyclone.

Table 1 Constant geometrical dimensions for cyclone.

\begin{tabular}{|c|c|c|c|c|c|c|c|c|}
\hline Parameter & $b$ & $d$ & $d_{1}$ & $D$ & $H_{\mathrm{t}}$ & $H_{2}$ & $H_{3}$ & $S$ \\
\hline Size $(\mathrm{mm})$ & 14 & 15 & 25 & 82 & 305 & 45 & 75 & 25 \\
\hline
\end{tabular}

To analyze the flow fields, four different inlet types are compared: case $1 \#$ with single inlet, cases $2 \#$ and 3\# with symmetric double-inlet for two different dimensions, case $4 \#$ with up-down doubleinlet. The detailed structural parameters are listed in Table 2 .

Table 2 Detailed parameters with four different inlet types for cyclone.

\begin{tabular}{|c|c|c|c|c|}
\hline Case & $1 \#$ & $2 \#$ & $3 \#$ & $4 \#$ \\
\hline Number of inlet & 1 & 2 & 2 & 2 \\
\hline$a(\mathrm{~mm})$ & 20 & 20 & 10 & 10 \\
\hline$c(\mathrm{~mm})$ & 61.5 & 61.5 & 61.5 & 61.5 \\
\hline$u(\mathrm{~m} / \mathrm{s})$ & 10 & 5 & 10 & 10 \\
\hline
\end{tabular}

Based on the results of inlet types, four different inlet lengths for cyclone are investigated. The detailed parameters are listed in Table 3. The geometries and operated parameters keep the same as case $3 \#$ except for changing the inlet length.

\subsection{Model description}

Considering the flow field feature of cyclone, the model of turbulent flow is described by the RSM. It 
Table 3 Detailed parameters with four different inlet lengths for cyclone.

\begin{tabular}{|c|c|c|c|c|}
\hline Case & $5 \#$ & $6 \#$ & $7 \#$ & $8 \#(3 \#)$ \\
\hline$D(\mathrm{~mm})$ & 82.00 & 82.00 & 82.00 & 82.00 \\
\hline$a(\mathrm{~mm})$ & 10 & 10 & 10 & 10 \\
\hline$c(\mathrm{~mm})$ & 26.83 & 41.00 & 51.25 & 61.5 \\
\hline $2 c / D$ & 0.654 & 1.000 & 1.250 & 1.5 \\
\hline
\end{tabular}

abandons the isotropic eddy-viscosity hypothesis and adopts the assumption of anisotropic (Li et al. 2020; Hreiz et al. 2011). The interface between the gas and water is tracked by the VOF model (Hwang et al. 2013; Lazrag et al. 2016), and the motions of particle are described by the DPM (Song et al. 2016; Safikhani and Mehrabian 2016). In the RSM, the transport equation can be determined as follows (Oh et al. 2015):

$\frac{\partial}{\partial t}\left(\rho \overline{u_{i}^{\prime} u_{j}^{\prime}}\right)+\frac{\partial}{\partial x_{k}}\left(\rho u_{k} \overline{u_{i}^{\prime} u_{j}^{\prime}}\right)=D_{T, i j}+P_{i j}+\varphi_{i j}-\varepsilon_{i j}$

where the two terms in the left side are the local time derivative of stress and convective transport, respectively. The four terms in the right side are:

the stress diffusion term:

$D_{i j}=-\frac{\partial}{\partial x_{k}}\left[\rho \overline{u_{i}^{\prime} u_{j}^{\prime} u_{k}^{\prime}}+\left(\overline{p^{\prime} u_{j}^{\prime}}\right) \delta_{i k}+\overline{\left(p^{\prime} u_{i}^{\prime}\right)} \delta_{j k}+\mu\left(\frac{\partial}{\partial x_{k}} \overline{u_{i}^{\prime} u_{j}^{\prime}}\right)\right]$ (1a)

the shear production term:

$P_{i j}=-\rho\left[\overline{u_{i}^{\prime} u_{k}^{\prime}} \frac{\partial u_{j}}{\partial x_{k}}+\overline{u_{j}^{\prime} u_{k}^{\prime}} \frac{\partial u_{i}}{\partial x_{k}}\right]$

the pressure strain term:

$\Pi_{i j}=p \overline{\left(\frac{\partial u_{i}^{\prime}}{\partial x_{j}}+\frac{\partial u_{j}^{\prime}}{\partial x_{i}}\right)}$

and the dissipation term:

$\varepsilon_{i j}=-2 \mu \overline{\frac{\partial u_{i}^{\prime}}{\partial x_{k}} \frac{\partial u_{j}^{\prime}}{\partial x_{k}}}$

In the VOF model, the tracking of interface between the phases is accomplished by the solution of a continuity equation for the volume fraction of one of the phases. For the $q$ th phase, this equation has the following form (Lazrag et al. 2016):

$\frac{1}{\rho_{\mathrm{q}}}\left[\frac{\partial}{\partial t}\left(\alpha_{\mathrm{q}} \rho_{q}\right)+\nabla\left(\alpha_{\mathrm{q}} \rho_{q} \overrightarrow{\mathrm{u}}_{\mathrm{q}}\right)\right]=S_{\alpha_{\mathrm{q}}}+\sum_{p=1}^{n}\left(m_{p q} m_{q p}\right)$

In this work, the interaction between particles is neglected since the inlet particle volume fraction of $2 \%$ is the diluted phase. Only the gravity and gas drag forces on particles are calculated in a Lagrangian reference frame, depicted as ( $\mathrm{Su}$ et al. 2011): $\frac{d \vec{u}_{p}}{d t}=F_{D}\left(\vec{u}-\vec{u}_{p}\right)+\frac{\vec{g}\left(\rho_{p}-\rho\right)}{\rho_{p}}$

$F_{D}=\frac{18 \mu C_{D} R e_{p}}{24 \rho_{p} d_{p}^{2}}\left(\vec{u}-\vec{u}_{p}\right)$

The drag coefficient $C_{\mathrm{D}}$ for spherical particles is calculated using the following correlations (Morsi and Alexander 1972):

$C_{D}=\left\{\begin{array}{lc}\frac{24}{R e} & R e_{P} \leq 1 \\ \frac{24\left(1+0.15 R e_{\mathrm{p}}^{0.687}\right)}{R e_{\mathrm{p}}} & 1<R e_{P} \leq 1000 \\ 0.44 & R e_{P}>1000\end{array}\right.$
$R e_{p}=\frac{\rho d_{p}\left|u_{p}-u\right|}{\mu}$

The particle trajectory is calculated by (Gao et al. 2020):

$\frac{d \vec{x}_{p}}{d t}=\vec{u}_{p}$

Discrete Random Walk (DRW) model is used to model the turbulent dispersion of particles (Song et al. 2017) (Abdulaziz and Shamsul 2020). After considering the anisotropy of Reynolds stress in the Reynolds stress model, the random pulsation velocity can be expressed as (Shafique et al. 2020):

$u^{\prime}=\varsigma \sqrt{\left(u^{\prime}\right)^{2}}$

$v^{\prime}=\varsigma \sqrt{\left(v^{\prime}\right)^{2}}$

$w^{\prime}=\varsigma \sqrt{\left(w^{\prime}\right)^{2}}$

$\varsigma$ is a random number subject to normal distribution. The amount under the root sign is the Reynolds stress of the local velocity fluctuation. The time scale:

$\tau_{e}=-T_{L} \log (r)$

\subsection{Boundary conditions}

The inlet boundary condition is velocity inlet and the direction of velocity is normal to the inlet. The calculation formula of selecting turbulent intensity and hydraulic diameter is as follows:

$I=0.16 \mathrm{Re}^{-1 / 8}$

(9)

$D_{h}=D$

The air volume flow rate is $10.08 \mathrm{~m}^{3} / \mathrm{h}$ for all cyclone, density of $1.225 \mathrm{~kg} / \mathrm{m}^{3}$ and dynamic viscosity of $1.7894 \times 10^{-5} \mathrm{~Pa} \cdot \mathrm{s}$ (Raeymaekers et al. 2007). Particles with density of $998.2 \mathrm{~kg} / \mathrm{m}^{3}$ are injected into the cyclone along with the air flow (Wilkes 2017). The velocity of particles is supposed 
to equal to the gas inlet velocity, and the diameter distribution of particles is supposed to obey the Rosin Rammler method. Five water droplets with different diameters were selected in the model, with the maximum diameter of $5 \mu \mathrm{m}$, the minimum diameter of $0.5 \mu \mathrm{m}$, and the average diameter of 2 $\mu \mathrm{m}$. Outlet of vortex finder is defined as the pressure outlet condition, and particles escape from the outlet. Underflow outlet and other boundaries are defined as no-slip wall condition and particles are assumed to be trapped by all walls.

\subsection{Numerical method}

The calculated model is considered to be threedimensional, double precision, adiabatic and viscous incompressible. The finite volume numerical method is employed to solve the governing equations with the fully implicit scheme. The presence of high pressure gradient and doublevortex flow structure needs an efficient algorithm for the pressure computation. The PRESTO pressure interpolation scheme is suitable in this field (Elsayed and Lacor 2011). The SIMPLEC algorithm is used for coupling between pressure and velocity (Song et al. 2017). The QUICK is employed to discrete convective terms in momentum and energy equations. The GeoReconstruct interpolation is applied to capture the variables on the gas-water interface (Oh et al. 2015) The Second-order upwind is used for the calculation of turbulent kinetic energy and turbulent dissipation rate (Demir et al. 2016). The first order upwind scheme is used for the discretization of the Reynolds stress equations. The convergence criterion is $10^{-4}$ for continuity, velocity components, RSM and liquid volume. A transient run was performed for VOF and DPM simulations with a time step of $2 \times 10^{-4} \mathrm{~s}$.

\subsection{Grid independent study}

The corresponding mesh of numerical model is shown in Fig. 2. The grid is generated using the commercial software ICEM and uniformly dispersed hexahedral elements for all simulations.

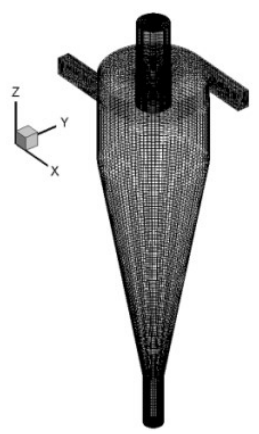

Fig. 2. Surface mesh for cyclone $3 \#$.

A grid independent study for case $3 \#$ is conducted after careful examination of the static pressure and total pressure. Four levels of uniform grids 91786, 141556,301564 and 350248 cells are investigated to validate the independence of grid size. The relative differences of static pressure and total pressure are given after refined grid number. The computational results of the four grid types are presented in Table 4. Results show that grid number 301564 is enough for all the subsequent calculations since the relative differences both of static pressure and total pressure are less than $1 \%$.

Table 4 Details of grid independent study.

\begin{tabular}{|c|c|c|c|c|c|}
\hline Total number of cells & 91786 & 141556 & 301564 & 350248 \\
\hline $\begin{array}{c}\text { Static pressure drop } \\
(\mathrm{Pa})\end{array}$ & 500.39 & \multicolumn{2}{|c|}{456.21} & 438.17 & 435.33 \\
\hline $\begin{array}{c}\text { Relative difference } \\
(\%)\end{array}$ & \multicolumn{2}{|c|}{9.6} & \multicolumn{2}{|c|}{4.12} & \multicolumn{2}{l|}{0.65} \\
\hline $\begin{array}{c}\text { Total pressure drop } \\
(\mathrm{Pa})\end{array}$ & 467.05 & 432.31 & 417.47 & 414.9 \\
\hline $\begin{array}{c}\text { Relative difference } \\
(\%)\end{array}$ & 8.0 & 4.20 & \multicolumn{2}{|c|}{0.62} \\
\hline
\end{tabular}

\section{RESULTS AND DISCUSSION}

\subsection{Model validation}

It is necessary to test the model validation to ensure the reliability of current research. This is done by comparing the simulated results of pressure drops, tangential and axial velocities with the experimental values (Hoekstra 2000), shown in Fig. 3. and Fig. 4. The maximum deviation of pressure drop is less than $10 \%$. Moreover, there is a similar hump-peak curve of the tangential and axial velocities at $\mathrm{Z}=$ $942.5 \mathrm{~mm}$ from the cyclone bottom $(\mathrm{Dx} / \mathrm{D}=0.5)$.

The simulated results meet the experimental velocity profile with underestimation of the tangential velocity and overestimation of the axial velocity. Taken into the experimental uncertainty and complexity of the turbulent swirling flow in the cyclone, the current results could be accepted.

\subsection{Effect of inlet type}

3.2.1 The variation of the flow property in the axial direction

Among three velocity components in the cyclone, the tangential velocity is the largest and most predominant component, which results in the centrifugal force required for particle separation (Safikhani and Mehrabian 2016). The axial velocity is the main source of short circuit flow and the back-mixing phenomenon, while the radial velocity is negligible (Bogodage and Leung 2015). Therefore, the CFD simulation on the timeaveraged static pressure, the tangential and axial velocity will be analyzed for four cyclones considering the effect of inlet types, shown in Fig. $5 \sim 7$.

Figure 5 shows that the static pressure curves have the shape of a $\mathrm{V}$ profile for all cyclones. The static pressure is rather similar at different positions for a cyclone. The value of static pressure almost equal for all cyclones except case $2 \#$, which is due to the 


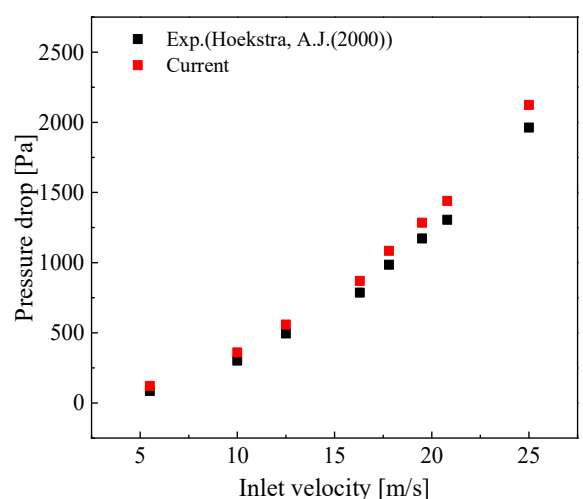

Fig. 3. Simulated and experimental results for pressure drops.
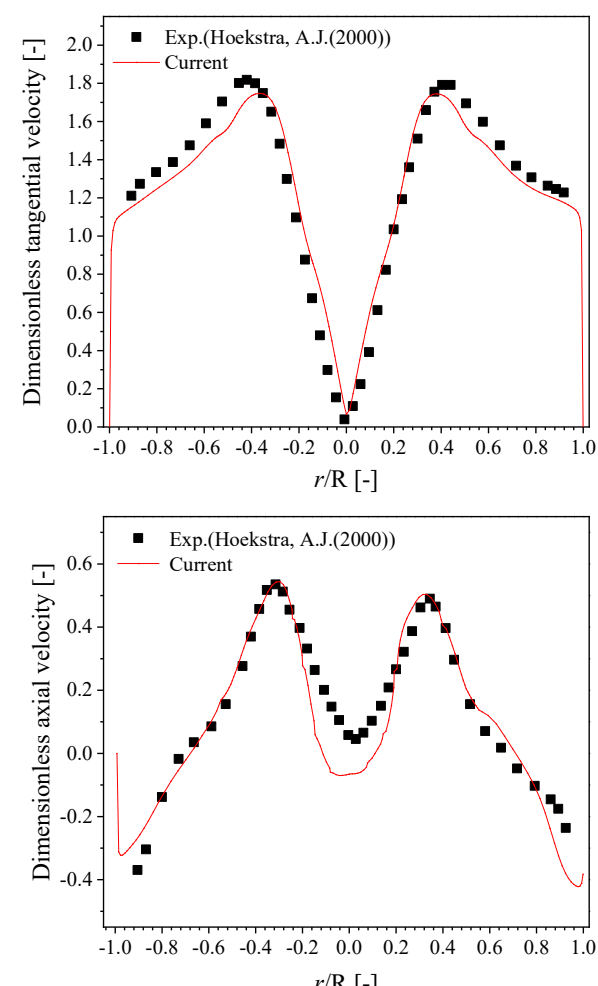

Fig. 4. Comparison of the tangential and axial velocities between simulated and experimental results $(Y=0, Z=942.5 \mathrm{~mm})$.

different inlet velocity caused by the inlet type. The same conclusion could be drawn from the tangential velocity distribution in Fig. 6. The tangential velocity profile for all cyclones displays typical Rankine vortex characteristic with inner quasi-force vortex and outer quasi-free vortex (Misiulia et al. 2015). It has the largest tangential velocity on each side and decreases to zero on the wall and in the center. Furthermore, the value variation of the maximum tangential velocity is rather limited at different positions, suggesting that no acceleration happens in the cyclone space. The effect of inlet types on the flow property could be obviously found in the axial velocity distribution in Fig. 7. It can be seen that axial velocity patterns have an inverted $\mathrm{W}$ profile for all cyclones except case $2 \#$
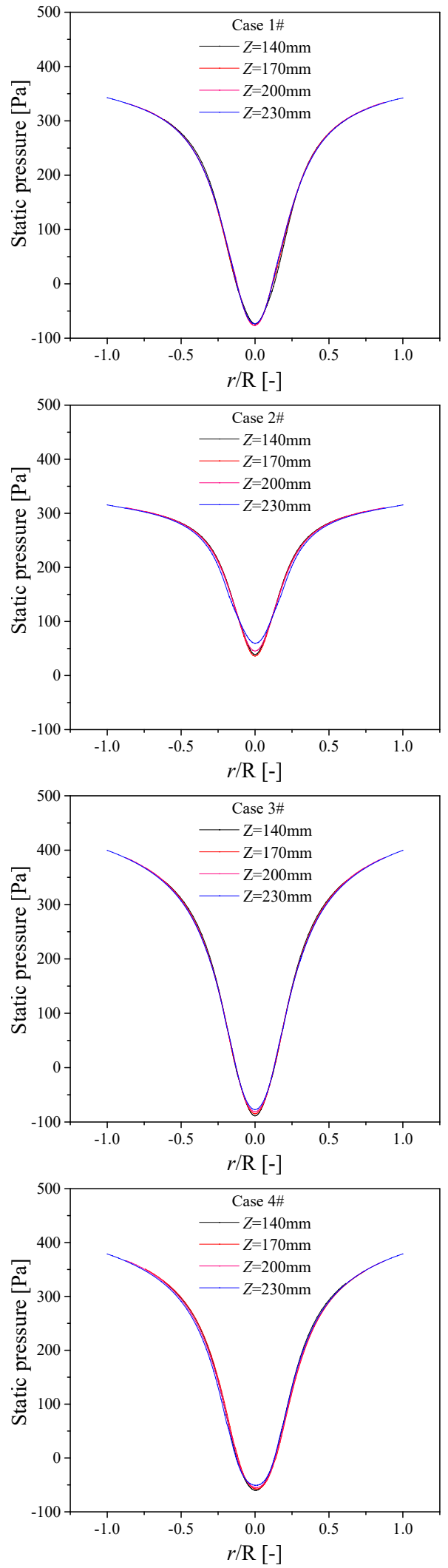

Fig. 5. Radial profile of static pressure.

with an inverted $\mathrm{V}$ profile due to the relatively weak negative pressure. Among all cases, case $3 \#$ shows the best axis-symmetry, which is beneficial for the stability of the flow field. 

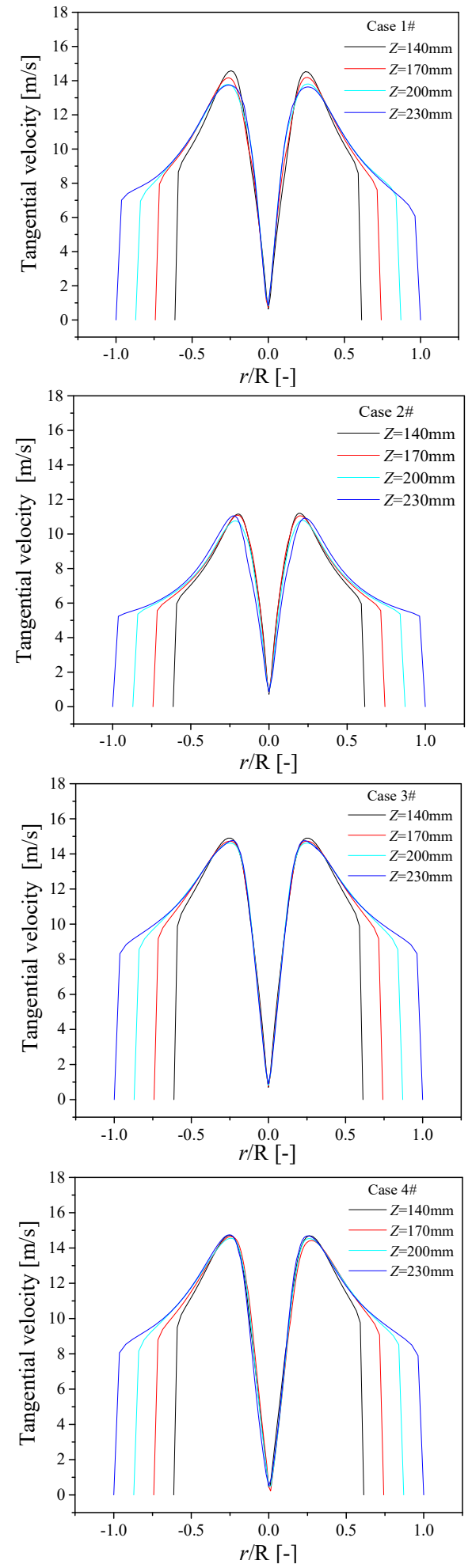

Fig. 6. Radial profile of tangential velocity.

\subsubsection{Flow stability}

Figure 8 shows the contours of $u_{x}$ velocity in the $X$ $\mathrm{Z}$ plane and $\mathrm{u}_{\mathrm{z}}$ velocity in the $\mathrm{X}-\mathrm{Y}$ plane for all cyclones. From Fig. 8(a), the symmetry of the flow is easily formed in the symmetric double-inlet of cases $2 \#$ and $3 \#$. The confusion degree in underflow pipe is very serious for asymmetric inlet cyclone
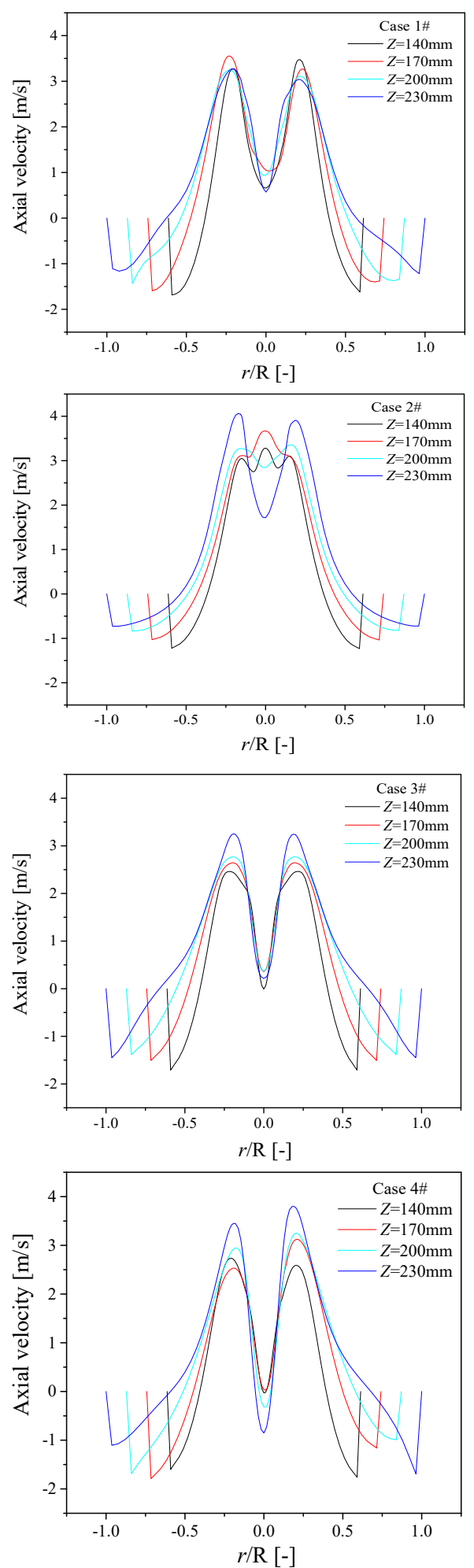

Fig. 7. Radial profile of axial velocity.

with cases $1 \#$ and $4 \#$, which will cause the backmixing phenomenon. From Fig. 8(b), the effect of inlet type on the flow stability begins at the inlet. The flow distribution of the asymmetric inlet cyclone is obviously irregular, in which the velocity is the highest at region $\mathrm{A}$ and the lowest at region $\mathrm{B}$. The unstable flow results in uneven shear force and some water droplets are carried into the internal 

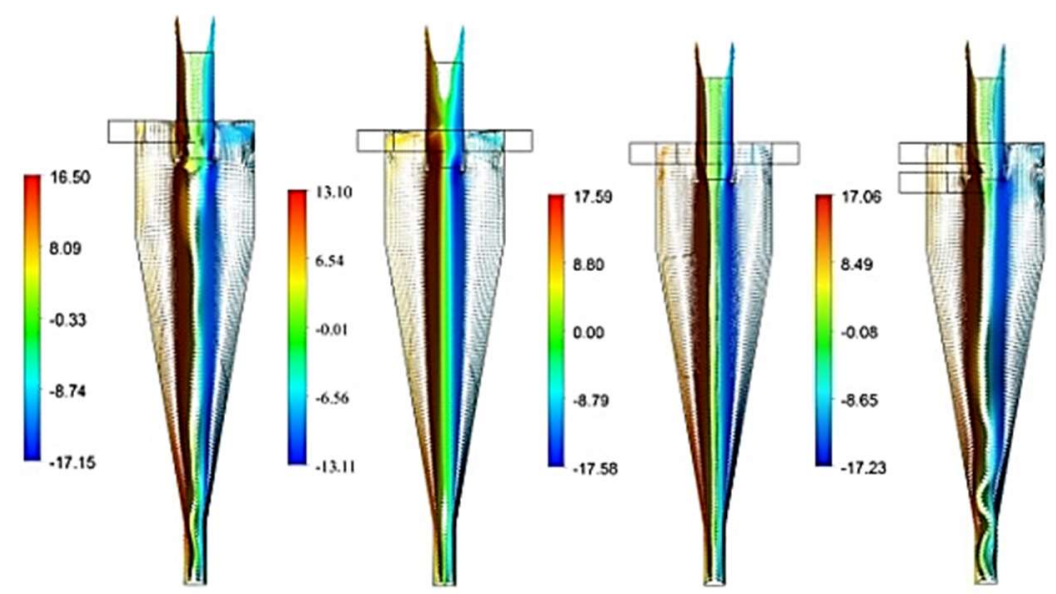

(a) $u_{x}$ vector profile of axial section

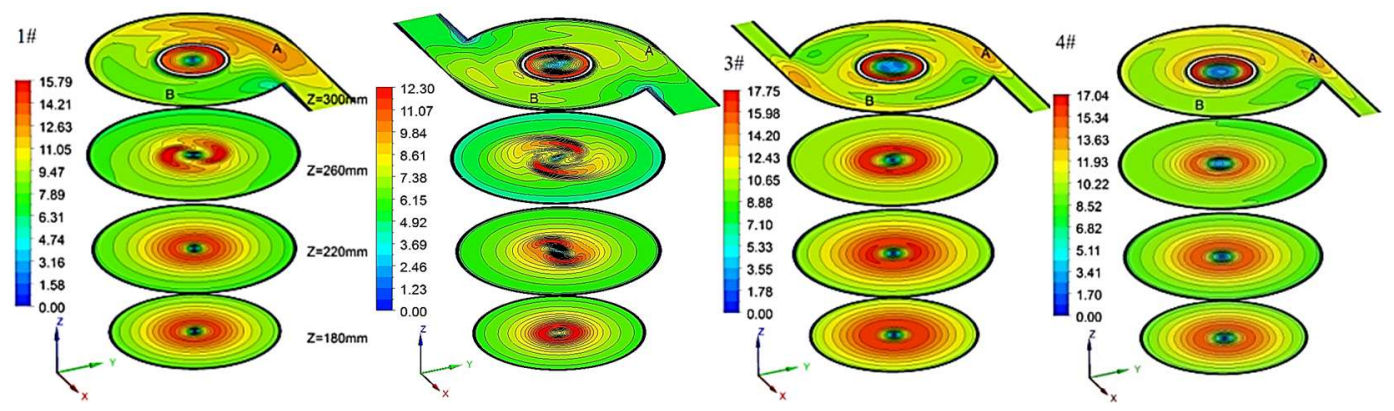

(b) $u_{z}$ vector profile of cross section

Fig. 8. Velocity profile of different inlet types $[\mathrm{m} / \mathrm{s}]$.

swirl. Therefore, the symmetric double-inlet of cyclone is the most appropriate inlet type.

\subsubsection{Secondary flow}

The interaction of the axial and radial velocity results in the secondary flow, which also has a significant effect on the separation performance. The secondary flow is described by the streamline in general, as shown at region A, B and C in Fig. 9, respectively. In region $\mathrm{A}$, the short-circuit flow is formed in the annular space of cyclone in the existent of the vortex finder. The inlet air flow firstly does a circular downward motion. Meanwhile, the air flow near the wall of vortex finder forms the low-speed laminar layer under the action of the frictional shears traction. When moving to the end of the vortex finder, it generates two kinds of flow. One flow with a high speed still keeps downward movement under the force of inertia. The other flow with a low speed would merge into central upward flow after consuming kinetic energy, which is the short-circuit flow. The latter flow usually carries a large number of particles and results in the low separation performance. The low speed flow is easy to form the short-circuit flow, which is the most serious in case $2 \#$ and not obvious in cases $1 \#$ and $3 \#$. In region $\mathrm{B}$, it presents an eddy flow feature, which is due to the formation of negative pressure in this place. The eddy flow makes the particles accumulate in region $\mathrm{B}$ and then decrease the separation efficiency. From Fig. 9. it could be found that all cyclones exist a degree of eddy flow, especially for cases $2 \#$ and $4 \#$. In region C, when the air flow with downward movement moves to the end of the cone section, it changes the motion direction and forms the upward swirl flow in the center of cyclones. However, a little air flow moves into the underflow tube and goes up again under the interaction of the upward swirl flow and the enclosed underflow tube. This flow causes an intensive momentum transfer and the energy loss, which is called the back-mixing flow. It appears the most serious in case $4 \#$ and could be almost neglected in case $3 \#$.

\subsubsection{Stability of the air-water interface}

Due to the existence of the back-mixing flow, the stability of the air-water interface needs to be further discussed, shown in Fig. 10. The red color represents air and the blue color represents water. It is obvious that the stability of the air-water interface of the symmetric inlets is better than that of asymmetric inlets. As expected, the stability of the air-water interface is broken for cases $1 \#$ and $4 \#$. There is an obvious shears breakage phenomenon at the air-water interface in a very short time. As times go by, some amounts of liquid water is carried to the separated region under the action of the upward air flow. It is the severe back-mixing phenomenon, 


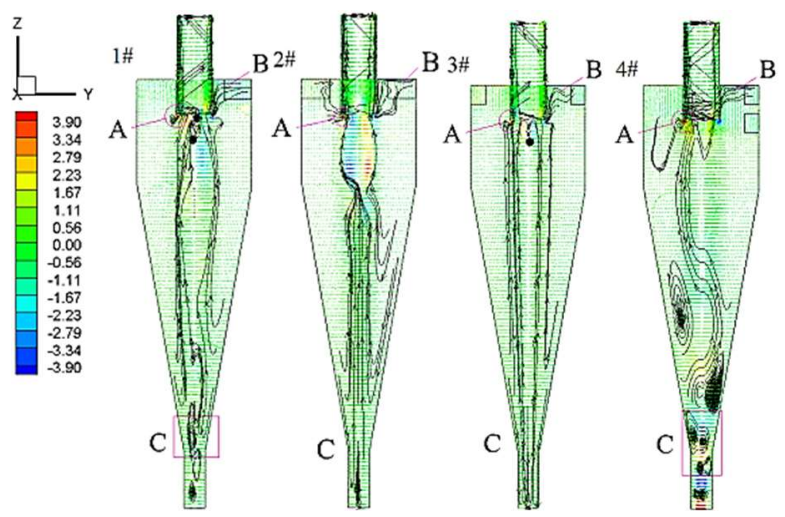

Fig. 9. Secondary flow (streamline) and radial velocity vector $[\mathrm{m} / \mathrm{s}]$.
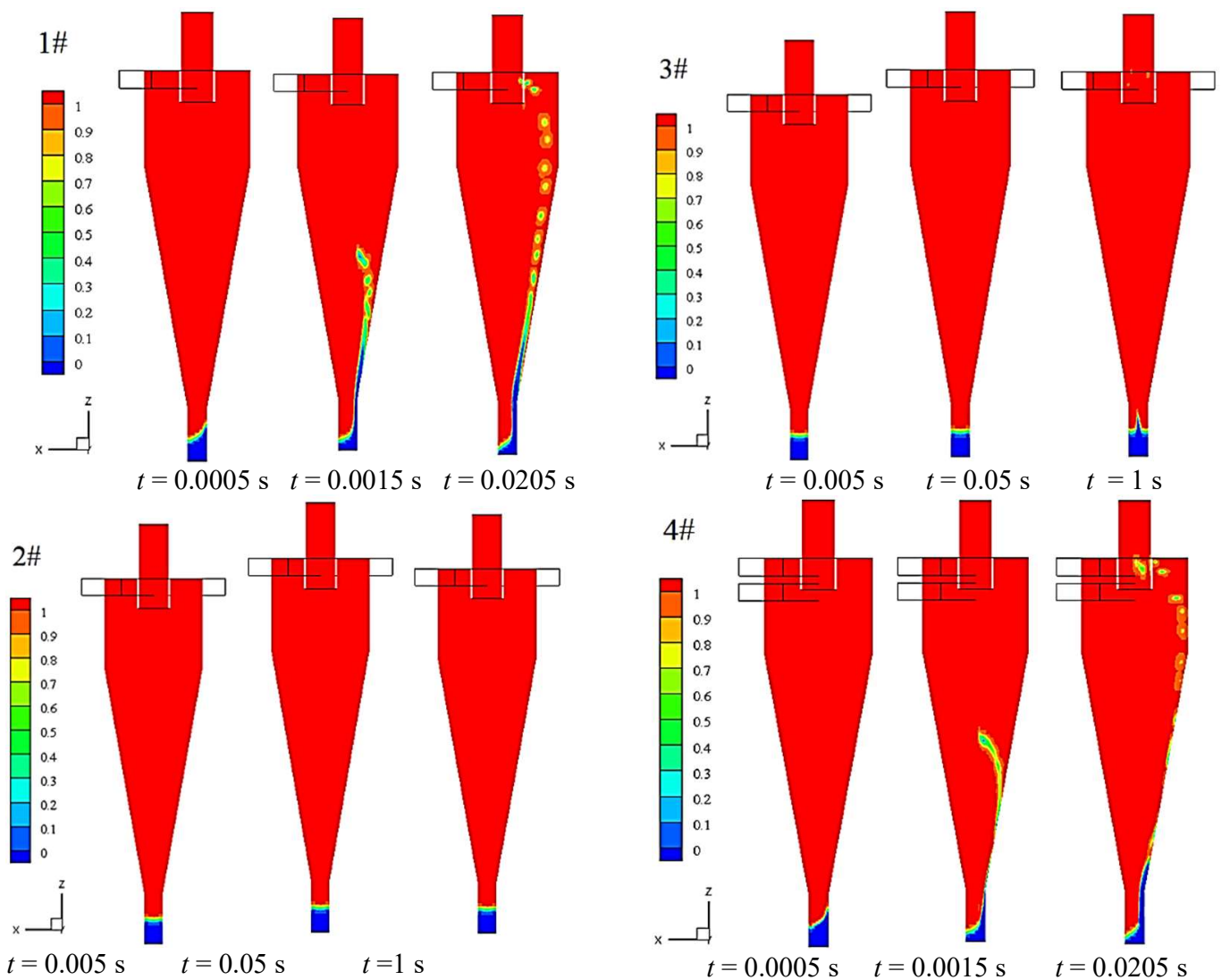

Fig. 10. Variety of the air-water interface.

which results in the decrease of separation performance. In cases $2 \#$ and $3 \#$, the air-water interface has good stability with the symmetric inlet. Because the high inlet velocity would increase the differential pressure between the central region and the near wall region and eventually lead to gas flow moving from the wall to the central, the air-water interface is slightly broken in case $3 \#$ at $\mathrm{t}=1 \mathrm{~s}$ compared with case $2 \#$.

\subsection{Effect of inlet length}

\subsubsection{Flow field}

Figure 11 shows the tangential velocity and the turbulent dissipation profile at $Z=300 \mathrm{~mm}$ for cases $5 \#, 6 \#, 7 \#$ and $8 \#$. The turbulent diffusion profile is plotted at section $\mathrm{A}^{\prime}-\mathrm{A}$ and viewed toward arrows. It is observed that the effect of inlet length on the flow field is very notable. The area of the highest tangential velocity enlarges with increasing the inlet length, which is beneficial to separate. The shorter the inlet length is, the bigger the turbulent dissipation is. It means there is a massive energy being wasted. In case $5 \#$, the air directly flows into the vortex finder instead of spinning down to the conical part, which will deteriorate the separation performance. When the inlet length increases to $1.25 \mathrm{D} / 2$, the area and intensity of the turbulent dissipation tend to be stable.

\subsubsection{Particle distribution}

Figure 12 shows the particle distribution for different inlet lengths after the flow field is steady. 


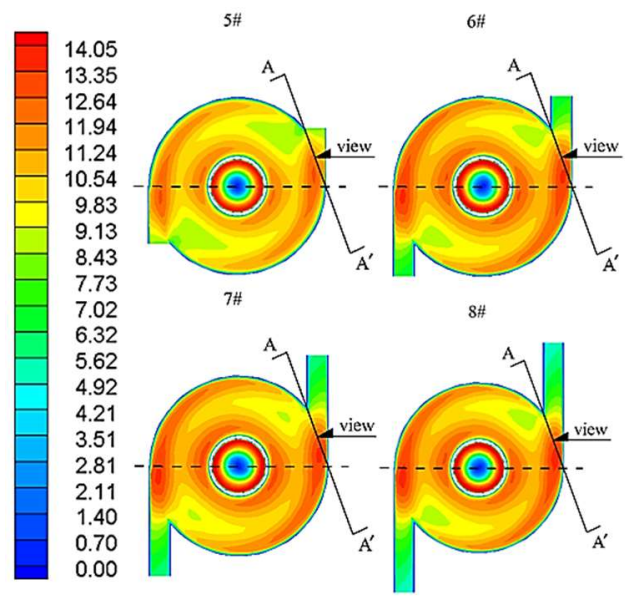

(a) Tangential velocity profile $[\mathrm{m} / \mathrm{s}]$

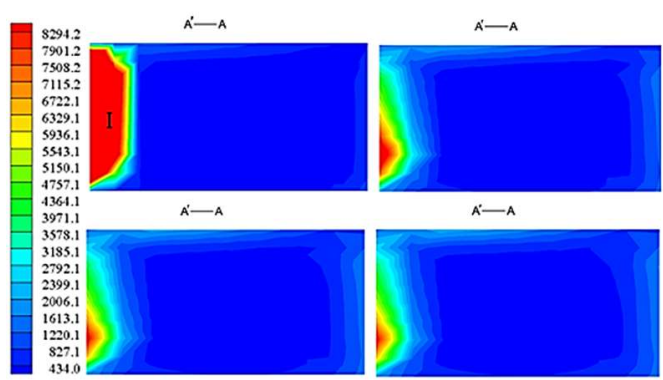

(b) Turbulent dissipation profile $\left[\mathrm{m}^{2} / \mathrm{s}^{3}\right]$

Fig. 11 Flow fields of different inlet lengths at $Z$ $=300 \mathrm{~mm}$.

Compared with other cyclones, the particles in case $5 \#$ appear an earlier tend to close to the vortex finder. It easily results to the particles escaping from the vortex finder and failing to separate. The particle behavior is almost the same in cases $6 \#$ and $7 \#$, which means the inlet length $c=1.25 \mathrm{D} / 2$ is enough.

\subsection{Separation efficiency}

The separation efficiency is a very important economical parameter for a cyclone. According to the above discussion on the inlet type and inlet length, the separation efficiency of the optimal cyclone case 3 \# was calculated. A DPM study has been performed by injecting the mixture of air and water from the inlet with a $10 \mathrm{~m} / \mathrm{s}$ velocity. The concentration and size of water is $2 \%$ and from 0.1 to $6 \mu \mathrm{m}$, respectively. The separation efficiency $\eta$ is defined as the following equation:

$$
\eta=1-\frac{N_{\text {escape }}}{N_{\text {tracked }}}
$$

As shown in Fig. 13, the separation efficiency increases with the increment of the particle diameter. When the particle diameter is larger than $5 \mu \mathrm{m}$, the complete separation could be realized. It is mainly because the larger the diameter of the particle is, the greater the centrifugal force is. The large particles will be successfully separated from the air in a short time. However, the small particles will escape from the vortex finder resulting in the separation failure.

\section{Conclusions}

In current work, a numerical study has been performed to examine the flow field characteristics of cyclones with different inlet types and inlet lengths. The findings are summarized as follows:

1) The cyclone with symmetric double-inlets could eliminate the unsteady flow field and the back mixing phenomena.

2) Increasing the inlet length of cyclone, the turbulent dissipation is reduced in the inlet, which is beneficial to enhance the separate efficiency. When the inlet length reaches to $1.25 \mathrm{D} / 2$, the area and intensity of the turbulent dissipation tend to be stable.

3) The optimum inlet of the cyclone is a symmetric double-inlet and an inlet length of $1.25 \mathrm{D} / 2$. When the particle diameter is larger than $5 \mu \mathrm{m}$, the complete separation could be realized.

This paper mainly studies the influence of inlet type and length of cyclone separator on its separation performance, without considering the limitation of cyclone separator shape and size on its installation. In the later stage, the influence of diameter length ratio on separation performance can be studied to reduce the volume of cyclone separator and improve separation efficiency.

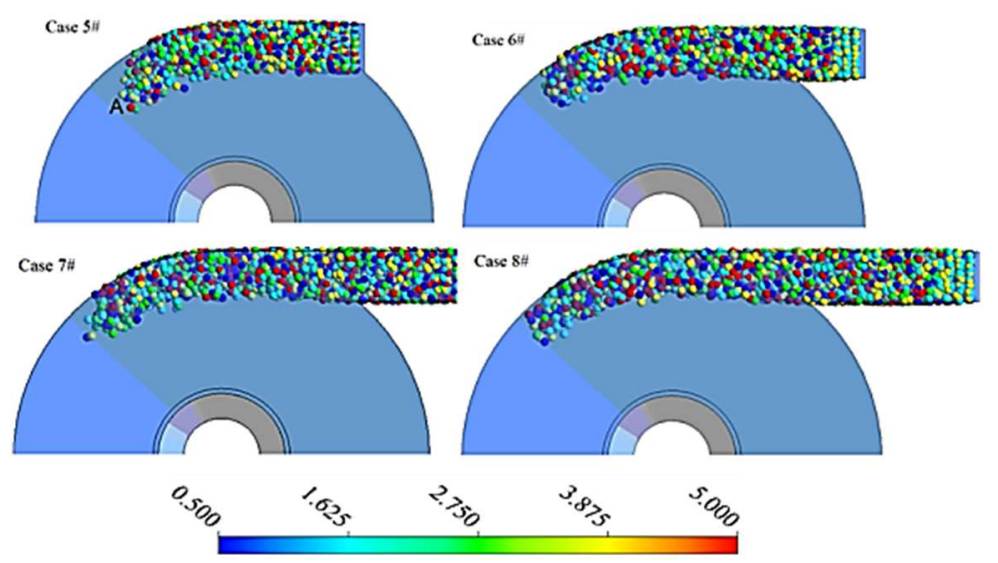

Fig. 12 Spatial distribution of different sized particles for different inlet lengths. 


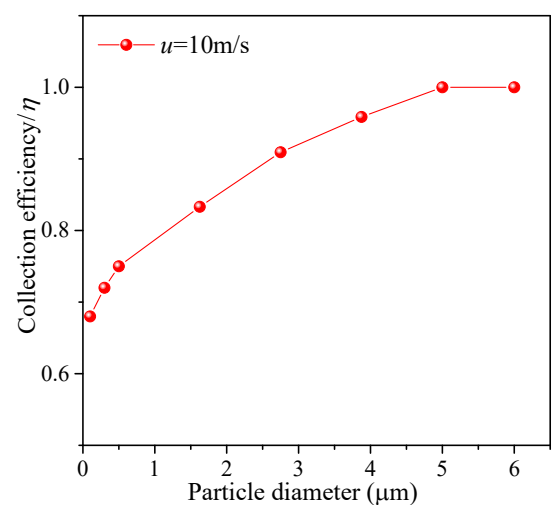

Fig. 13. Separation efficiency for cyclone $5 \#$.

\section{ACKNOWLEDGEMENTS}

The work was supported by the National Nature Science Foundation of China (Grant No. 51206071), the Hunan Provincial Natural Science Foundation of China (Grant No. 2019JJ40284, 2018JJ4054).

\section{REFERENCES}

Abdulaziz, G. A. and Q. Shamsul (2020). Effect of temperature variations on non-equilibrium and non-isothermal two-component liquid chromatography in cylindrical columns. Journal of Liquid Chromatography \& Related Technologies 43(19-20), 890-912.

Bao, L. L., Z. L. Liu and J. Liu (2017). Experimental study for the effect of the built-in cyclone configuration on the dehydration performance of the new wet-gas recycling supersonic separator. Science Technology and Engineering 17, 177-180.

Bogodage, S. G. and A. Y. Leung (2015). CFD simulation of cyclone separators to reduce air pollution. Powder Technology 286, 488-506.

Caliskan, M. E., I. Karagoz, A. Avci and A. Surmen (2019). An experimental investigation into the particle classification capability of a novel cyclone separator. Separation and Purification Technology 209, 908-913.

Demir, S., A. Karadeniz and M. Aksel (2016). Effects of cylindrical and conical heights on pressure and velocity fields in cyclones. Powder Technology 295, 209-217.

Elsayed, K. (2015). Optimization of the cyclone separator geometry for minimum pressure drop using Co-Kriging. Powder Technology 269, 409-424.

Elsayed, K. and C. Lacor (2011). Numerical modeling of the flow field and performance in cyclones of different cone-tip diameters. Computers \& Fluids 51(1), 48-59.

Gao, Z., J. Wang, Z. Liu, Y. Wei and Y. Mao (2020). Effects of different inlet structures on the flow field of cyclone separators. Powder
Technology 372, 519-531.

Hoekstra, A. J. (2000). Gas flow field and collection efficiency of cyclone separators. Thesis. Delf University of Technology, 2000. Hoekstra, A. $\mathrm{J}$

Hreiz, R., C. Gentric and N. Midoux (2011). Numerical investigation of swirling flow in cylindrical cyclones. Chemical Engineering Research and Design 89, 2521-2539.

Hwang, K. J., Y. W. Hwang and H. Yoshida (2013). Design of novel hydrocyclone for improving fine particle separation using computational fluid dynamics. Chemical Engineering Science $85,62-68$.

Kuznetsov, B. N. and M. L. Shchipko (2010). Processing of the Kansk-Achinsk Brown Coal into Synthetic Fuel. Chemistry for Sustainable Development 18, 239-251.

Lazrag, M., D. L. Mejia-Mendez, C. Lemaitre, P. H. E. Stafford, R. Hreiz, R. Privat, A. Hannachi and D. Barth (2016). Thermodynamic and hydrodynamic study of a gas-liquid flow in a cyclone separator downstream supercritical drying. The Journal of Supercritical Fluids $118,27-38$.

Shafique, S., M. Afzal and R. Nawaz (2020). On the attenuation of fluid - structure coupled modes in a non-planar waveguide. Mathematics and Mechanics of Solids 25(10), 108128652091144

Li, Z., Z. Wang and J. Shi (2020). Optimization design of the integral inertial particle separator based on response surface method. Journal of Applied Fluid Mechanics 13(1), 133-145.

Morsi, S. and A. Alexander (1972). An investigation of particle trajectories in twophase flow systems. Journal of Fluid Mechanics 55(2), 193-208.

Misiulia, D., A. G. Andersson and T. S. Lundström (2015). Effects of the inlet angle on the flow pattern and pressure drop of a cyclone with helical-roof inlet. Chemical Engineering Research and Design 102, 307-321.

Nardo, A. D., G. Calchetti and S. Stendardo (2018). Modeling and simulation of an oxygen-blown bubbling fluidized bed gasifier using the computational particle- fluid dynamics (cpfd) approach. Journal of Applied Fluid Mechanics 11(4), 825-834.

Oh, J., S. Choi and J. Kim (2015). Numerical simulation of an internal flow field in a uniflow cyclone separator. Powder Technology 274, 135-145.

Pan, J., Q. Shen, X. Cui, J. Wu and H. Wang (2021). Cyclones of different sizes and underflow leakage for aerosol particles separation enhancement. Journal of Cleaner Production $280,124379$.

Prabhansu, S., Rajmistry, S. Ganguli, P. Chandra 
S. Wu et al. / JAFM, Vol. 15, No. 2, pp. 591-601, 2022.

and P. K. Chatterjee (2017). Numerical Analysis of Gas-Solid Behavior in a Cyclone Separator for Circulating Fluidized Bed System. Journal of Applied Fluid Mechanics 10(4), 101167-1176.

Prasanna, N., K. Subramanian, S. Ajay, T. Rajagopal and V. Vigneshwaran (2021). CFD study on the performance of reducing pressure drop holes in cyclone separator. Materials Today: Proceedings 43, 1960-1968.

Rafiee, S. E. and M. M. Sadeghiazad (2016). Efficiency evaluation of vortex tube cyclone separator. Applied Thermal Engineering 114, 300-327.

Raeymaekers, B., I. Etsion and F. E. Talke (2007). Enhancing tribological performance of the magnetic tape/guide interface by laser surface texturing. Tribology Letters 27, 89-95.

Safikhani, H. and P. Mehrabian (2016). Numerical study of flow field in new cyclone separators, Advanced Powder Technology 27(2), 379-387.

Song, C., B. Pei, M. Jiang, B. Wang, D. Xu and Y. Chen (2016). Numerical analysis of forces exerted on particles in cyclone separators. Powder Technology 294, 437-448.

Song, J., Y. Wei, G. Sun and J. Chen (2017) Experimental and CFD study of particle deposition on the outer surface of vortex finder of a cyclone separator. Chemical Engineering Journal 309, 249-262.
Su, Y., A. Zheng and B. Zhao (2011). Numerical simulation of effect of inlet configuration on square cyclone separator performance. Powder Technology 210, 293-303.

Wang, D. (2004). Dust collector with an inbuilt cyclonic water filter. US20040139711.

Wang, S., H. Li, R. Wang, W. Xu and Q. Sun (2019). Effect of the inlet angle on the performance of a cyclone separator using CFD-DEM. Advanced Powder Technology 30, 227-239.

Wasilewski, M. and L. S. Brar (2019). Effect of the inlet duct angle on the performance of cyclone separators. Separation and Purification Technology 213, 19-33.

Wilkes, J. O. (2017). Fluid Mechanics for Chemical Engineers: with Microfluidics, CFD, and COMSOL Multiphysics 5, 3rd Edition.

Wright, H. A., B. Demirel, S. Mohedas, R. Bahman, R. Huang, D. Ferous, J. W. Taylor, D. J. Duvenhange and S. Rolfe (2013). Protected fischer-tropsch catalyst and method of providing same to a fischer-tropsch process. US.

Yao, Y. G., W. S. Huang and Y. X. Wu (2021). Effects of the inlet duct length on the flow field and performance of a cyclone separator with a contracted inlet duct. Powder Technology 393, 12-22. 